\title{
Monitoring network-based infrastructure for forest fire detection
}

\author{
V. S. Kharchenko ${ }^{1}$, A. A. Orekhov ${ }^{1}$, D. A. Kotchkar ${ }^{2}$ \\ \& V. V. Bogomolov ${ }^{3}$ \\ ${ }^{1}$ National Aerospace University, KhAI, Ukraine \\ ${ }^{2}$ Research and Development Company Lesinform, Ukraine \\ ${ }^{3}$ Research Institute of Forestry and Forest Amelioration, Ukraine
}

\begin{abstract}
Information technology for design and reengineering of ground-based monitoring systems' infrastructure was developed for forecasting and early detection of forest fires based on wireless networking, video surveillance and sensor networks. The technology is based on the methods of two-dimensional and three-dimensional coverage of multi-colored geometric objects, considering placement points and areas of surveillance, sensors, and network infrastructure as a whole. Heuristic procedures of a spatial covering of multicolor flat figures for various configurations are offered. Instrumental means of design and reengineering of ground-based monitoring systems, as well as a decision-making support, was developed to detect ignition centers and ensure fire-fighting.
\end{abstract} Keywords: monitoring, forest fire, video, sensor networks, coverage, design.

\section{Introduction}

\subsection{Motivation}

Every year there are up to 400,000 forest fires occur on Earth, damaging about $0.5 \%$ of the total forest area and discharging millions of tons of products of combustion into the atmosphere. Some of them turn into catastrophic ones [1]. In the last decade the problem of forest fires has become particularly acute due to the sharp deterioration of the ecological environment.

Monitoring for forest fires is a system for observation and control of fire hazards in the forest under the terms of the weather, a condition of forest 
combustible materials, sources of fire and forest fires with a goal of timely development and implementation of measures to prevent forest fires and reduce damages.

Forecasting of forest fires can be made, on the basis of the analysis of monitoring data as a definition of a probability of the wildfires in time and space. Source data for the forest fire prediction are: the class of fire danger in woods in terms of weather; location and a forest area; terrain data; the existence of potential sources of fire, etc.

Modern systems of prevention and protection from forest fires should provide real-time monitoring for timely and optimal solutions. One possible solution is ground monitoring systems (GMS).

These systems are based on various technologies (video monitoring, sensor network, mesh networking, etc.). It is therefore important to implement a rational choice and sound integration of such technologies.

\subsection{Video and sensor network-based monitoring technologies analysis}

In order to ensure the timely forest fire detection GMS use observation towers. Construction and placement of the towers is based on the terrain, considering that their infrastructure could provide maximum overview of protected territory, timely detect emerging fires and identify fire locations with 2 or 3 towers. Currently the wireless video monitoring technologies are widely developed $[2$, 3]. Information from video cameras, installed in forests, is transmitted via radio channel to a uniform control station (with the possibility of remote monitoring by video cameras). After positioning the source of ignition, the entire video is stored on the server for further analysis and decision to start fire-fighting activities.

Recent achievements in wireless sensor networks indicate that they are a promising technology to establish GMS [4-7]. Modern sensors can detect and monitor a variety of static and dynamic parameters, including temperature, relative humidity, smoke, wind direction and speed. Sensor systems can be useful not only for fire detection, but also to take a decision on its liquidation. If the sensor network nodes are integrated with GPS or have their predefined location coordinates, it is possible not only to learn about the outbreak of fire, but also to determine accurately where the source of fire is.

Existing protocols have made nodes with sensors able to organize a network independently without customization.

The network infrastructure that combines a wireless network system of video monitoring (WNVM) and wireless sensor network (WSN) is a very promising solution for GMS. 


\section{Problem of integrated monitoring system development}

\subsection{Infrastructure of integrated ground-based monitoring systems}

The described architecture of integrated ground-based monitoring system is shown in Figure 1.

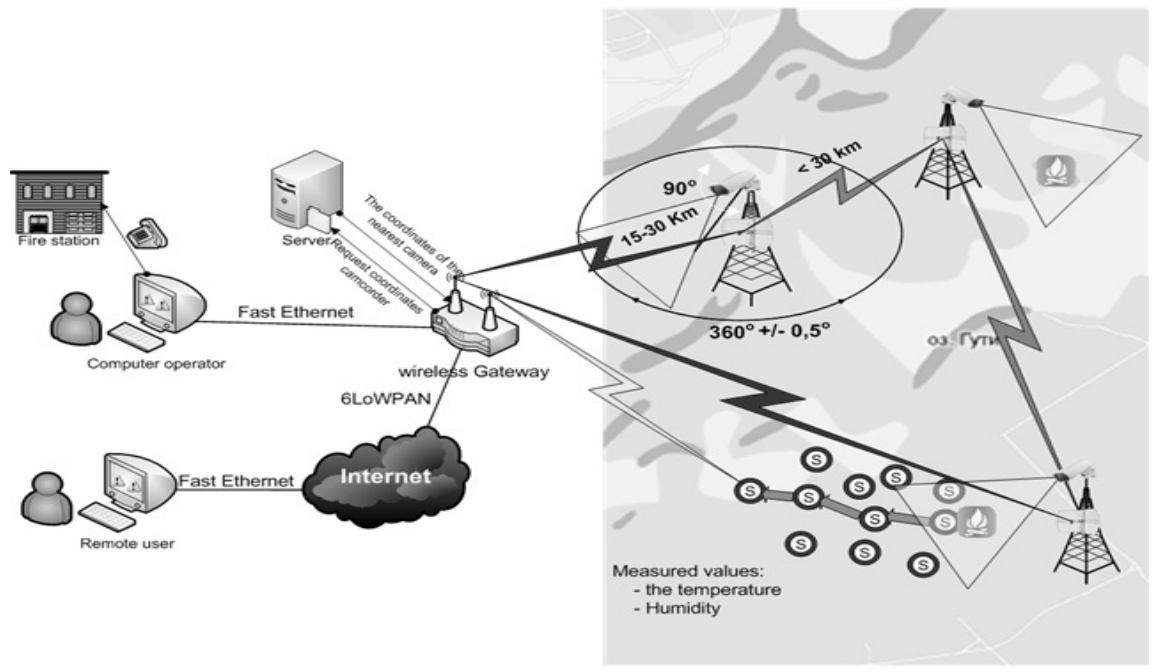

Figure 1: Infrastructure of the ground-based monitoring system for forest fires.

It is proposed to combine sensors with IP cameras to detect and verify fire [8]. When the sensor detects a fire, it sends an alarm signal to server via a wireless network. Software application of the server selects the cameras nearest to the sensor and turns them to the sensor, which raised the alarm. The video camera transfers images in real time and allows firefighters to confirm the existence of fire and avoid false alarms. WSN can be connected to the Internet via gateway.

Thus, the actual problem to create ground-based systems for monitoring is the choice of infrastructure based on various technologies which unites both WNVM and WSN options. Criteria for selecting the GMS infrastructure could be full control, timeliness, reliability and economic efficiency.

Development of specialized systems for Computer Aided Design (CAD GMS) and decision support systems (DSS GMS) is required to support the process of designing modern network infrastructures.

The objective of this work is to develop the cost-effective information technology to support design and reengineering of GMS, taking into account the existing infrastructure equipment characteristics of forest areas, natural limitations, etc. 


\subsection{Work related analysis}

The problems of designing and reengineering of the ground video-monitoring systems (GVMS) for detection of forest fires have been formulated in [3]. These objectives are the selection and/or placement of fire towers, as well as the selection of necessary equipment (video cameras, sensors, data transmission, etc.) to provide the required full control and minimal cost of the monitoring system. It is necessary to take into account the high fire danger zones and zones of limited control.

The problem of the optimal placement of towers and equipment may be formulated as the coverage problem. Covering with circles of the same radius was first considered in [9]. Today it has been used in sensor networks [10]. Combinations of circles with several different radii are possible. One approach to solving this problem as a problem of cutting and packing is given in [11].

\section{Methods for solving coverage problems}

Mathematical models for solving coverage problems can be represented by twoand three-dimensional multicolored geometric shapes with known configuration. All objects in the analysis are presented in the form of flat shapes - polygons. Figure 2 shows a model of a forest object, considering the class of fire hazard for individual polygons.



Figure 2: $\quad$ Model of the fire hazard polygons of the forest object.

A model, shown in figure 3, takes into account the terrain and visibility zones. Exact and heuristic methods are used to solve the coverage problem. Heuristic methods have been used widely due to the complexity of the exact methods. Covering procedure is given in [4]. The result of the procedure application to a model, defined as a two-dimensional multicolored geometric shape, is shown in Figure 4. 


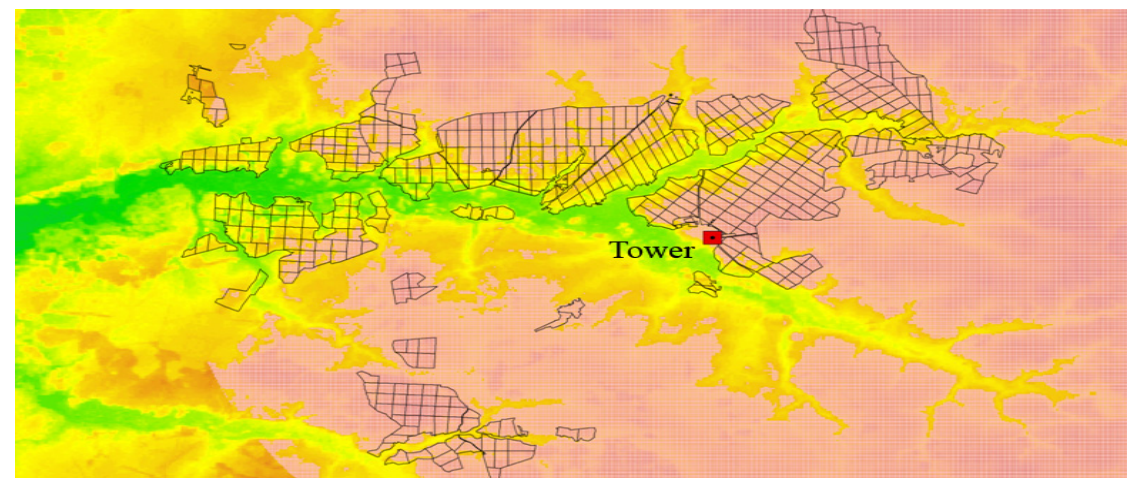

Figure 3: Model of the forest object based on topography and the fire tower visibility zones.



Figure 4: The area is covered by circles of small radius with regard to forbidden sites for sensor networks.

\section{Tools for GMS development and decisions making}

\subsection{System design automation}

Models and methods (below) were used to design tools for system design automation and decision support. Figure 5 shows the architecture of CAD GMS.

Design of GMS is implemented in two phases: creation of the cartographic basis and design itself.

To create cartographic basis a bitmap is loaded further digitizing. During digitizing the boundaries of analyzed objects are defined and the following characteristics are set up:

-border controlled territory;

-set of forbidden sites; 
-properties of the digitized object;

-characteristics of the forest;

-requirements to the monitoring system design.

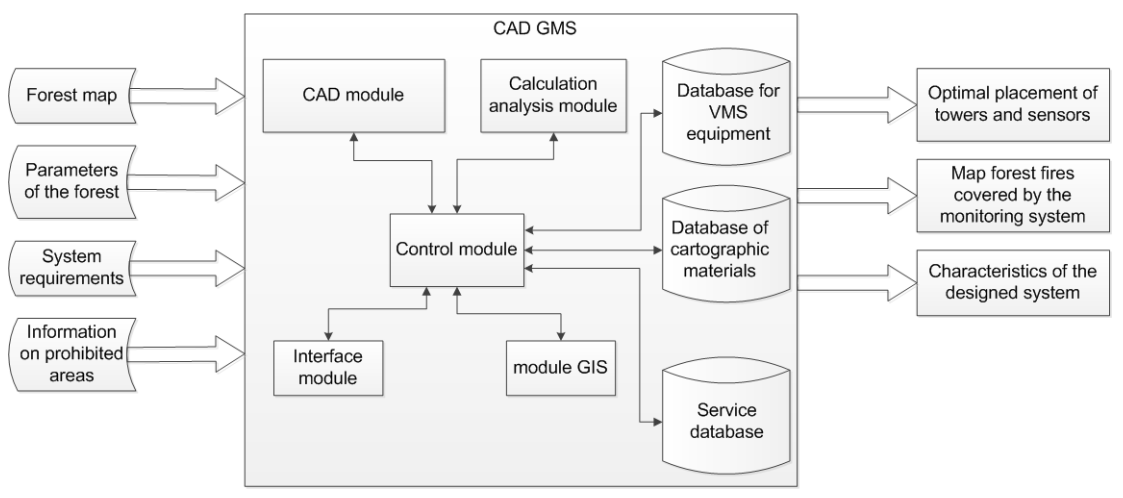

Figure 5: Architecture of CAD GMS.

At the design stage CAD GMS provides the following features:

- automatic coverage of the pre-defined parameters without existing towers;

- automatic coverage of the pre-defined parameters with existing towers;

- placement of equipment (towers, video, sensors);

- calculation of parameters of the designed monitoring system;

- calculation of the monitoring system cost.

The interface of CAD GMS is given Figure 6, showing the coverage for selected infrastructure (towers and equipment) of the designed monitoring system.

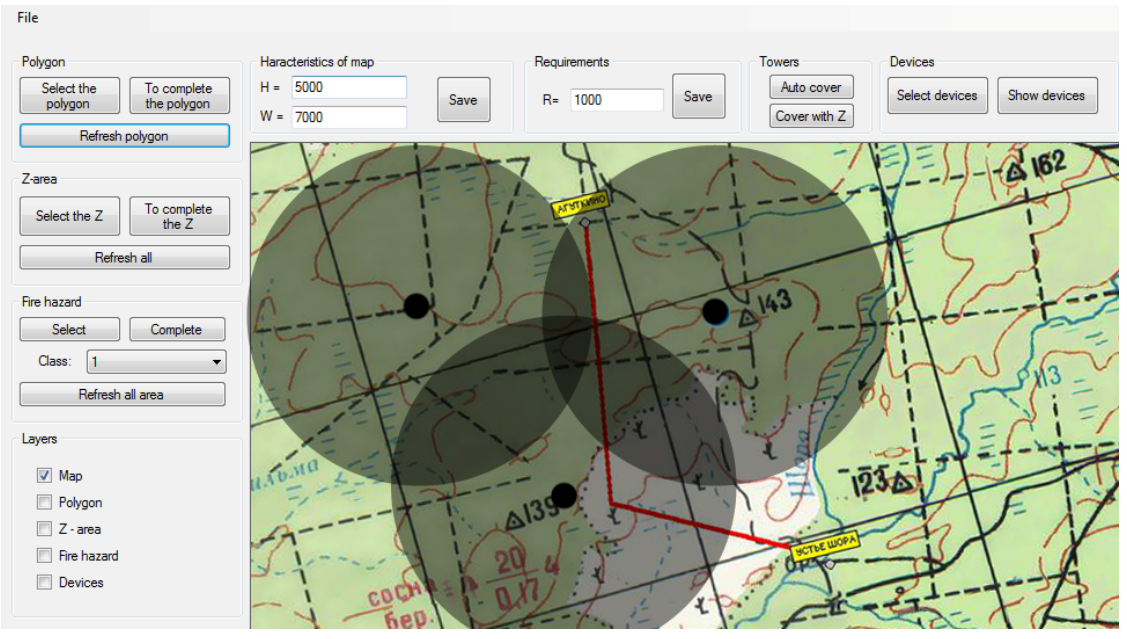

Figure 6: Monitoring system infrastructure. 
CAD GMS is implemented in C \# in Microsoft Visual Studio 2010. Silverlight technology was used to develop system's interface, allowing it to run applications that contain animation, vector graphics and audio-video information. The database is implemented on Microsoft SQL Server 2008.

Application programming interface ArcGIS API for Microsoft Silverlight / WPF was used to work with cartographic material (creation and digitization). This software allows you to create applications, using cartography, geo-coding and geo-processing provided by ArcGISServer and Bing services.

\subsection{Placing the observation tower with a view of the terrain}

Placement of the observation towers and definition of zones of visibility based on topography, these problems are implemented in a specialized module called Azimut-Pro.

The module has the following functions for placing observation towers:

- ability to add and delete towers, as well as make and adjust their characteristics (number, name, height above the ground, the absolute height of the tower grounds, zero reference);

- to calculate area of tower direct visibility;

- to enable or disable the satellite images;

- to take into account the direction of the tower's zero reference in the derivation of the line of sight;

- to move from a list of forest areas and quarters for displaying them in a cartographic window.

Figure 7 shows the window of the application in an append mode.

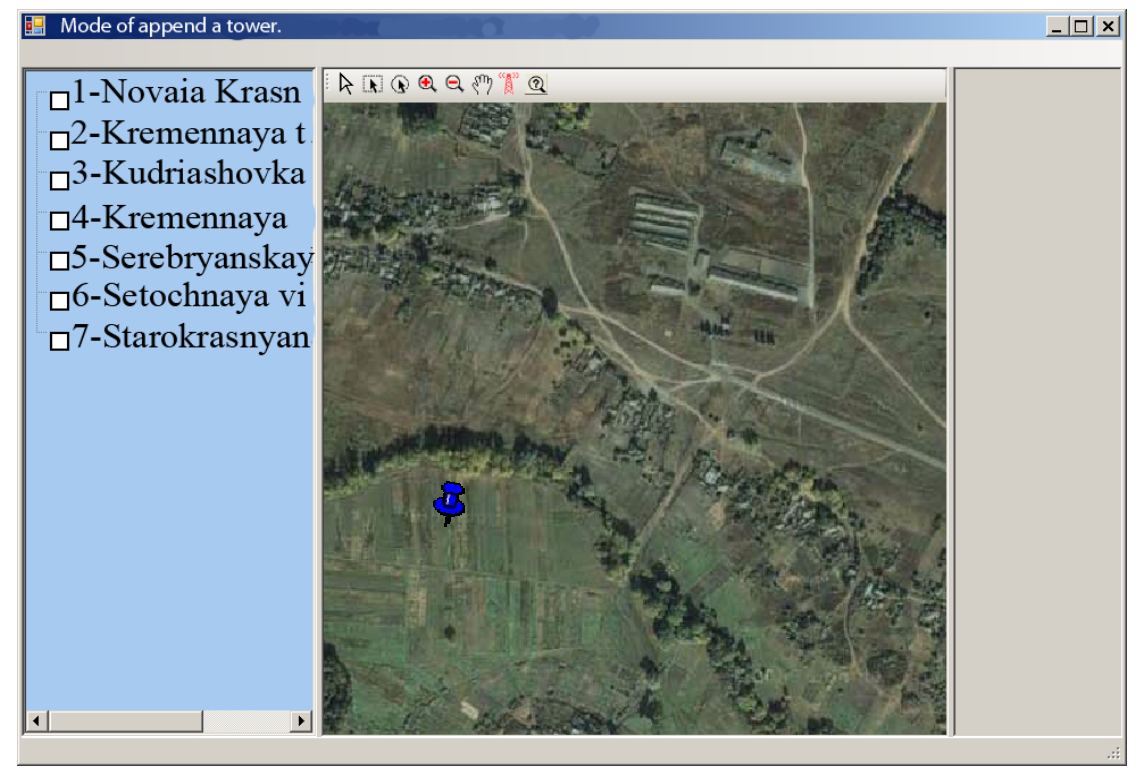

Figure 7: Operation of the Azimut-Pro module in the mode of appending a tower. 


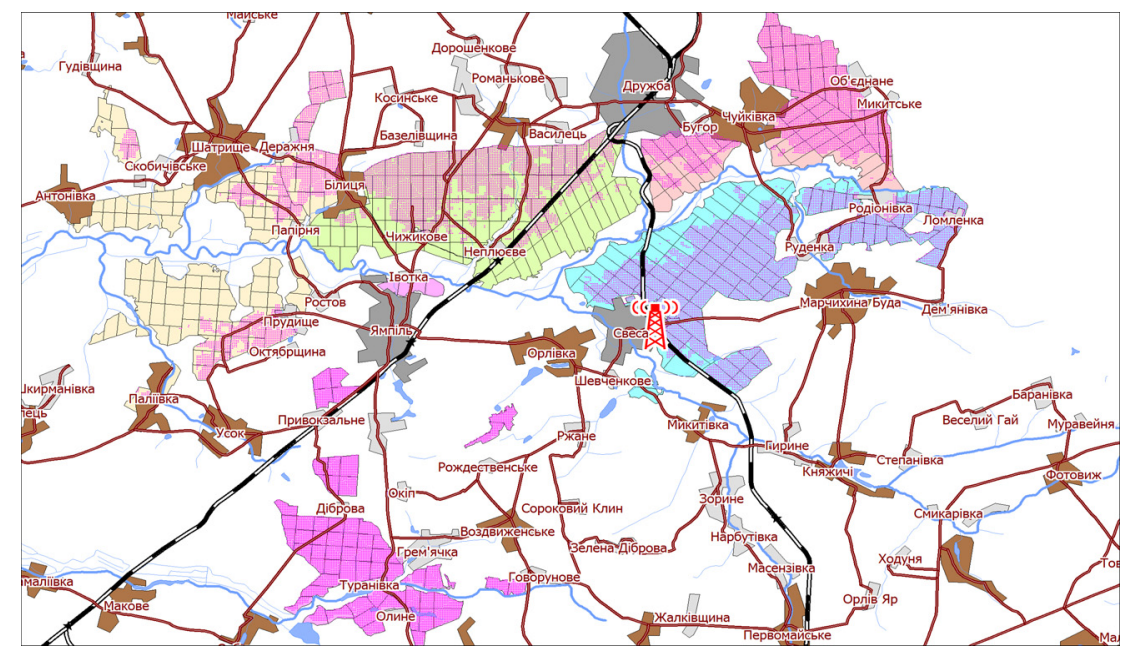

Figure 8: Calculation of the territory of the tower visibility based on topography.

Having defined coordinates, height of a tower and taking into account digital elevation model its visibility can be calculated (figure 8).

Functions of the decision-making support system DSS GMS are implemented in module Azimut-Pro, which is also intended to contain fire places (forest quarter coordinates) as seen from the fire towers. The program has features, allowing it to perform the surveillance towers placement considering the terrain.

Azimut-Pro program is created for employees of the forest protection service of forest enterprise. Time of fire extinguishing consists of the time, when exact location of fire was detected, and time to mobilize personnel and fire extinguishing equipment.

The ignition location is determined by the azimuthal measurements, performed with a video camera or a compass installed on fire tower. Water supply routes to fire-fighting vehicles can be defined by map layers.

The scope of Azimut-Pro includes the following map layers:

- polygons blocks

- ranges available;

- human settlements;

- hydrography (rivers and reservoirs);

- road network;

- orthophotoplanes or satellite images.

Determination of territory occupied by fire is made from the measurements of azimuths of the two towers. When observation is just visual an azimuth is measured with a compass. In this case we are dealing with magnetic azimuth, and to orient the direction correctly you need to take into account a magnetic declination of a given area. If you use video control system, camera zero reading is oriented to the north along the true meridian, so the azimuth reading is taken without amendment. 


\section{Conclusion}

In this paper we proposed the infrastructure of the network system for forest fires monitoring, as well as the method and tools for the design and decision-making ground monitoring systems.

In our consideration, integrated GMS-based video and sensor technologies are useful for monitoring and decision-making support to extinguish fires in relatively small forest areas with complex configuration. We are planning to continue the research in the following areas:

- improvement of covering techniques tailored to sensors and video cameras, and their consistent use;

- CAD and decision-making support system modernization based on multiparametric sensors.

\section{References}

[1] European Commission. Forest Fires in Europe 2009, Publications Office of the European Union: Luxembourg, 2010.

[2] Automatic Early Warning System for Forest Fires. FireWatch. www.fire-watch.de/cms

[3] Kharchenko, V.S., Orekhov, A.A., Medintsev, S.Yu., Kotchkar, D.A. \& Bogomolov, V.V, Ground video-monitoring systems for early detection of forest fires. Journal of information, control and management systems, 8(2), University of Zilina: Slovakia, pp.111-120, 2010.

[4] Hefeeda, M. \& Bagheri, M., Forest fire modeling and early detection using wireless sensor networks. Ad Hoc \& Sensor Wireless Networks, 7, pp.169224, 2009.

[5] Pripuzic, K., Early forest fire detection with sensor networks: sliding window skylines approach (Part 1). Proc. of the $12^{\text {th }}$ Int. Conf. KES 2008, eds. Belani, H. \& Vukovic, M.: Zagreb, pp.725 - 732, 2008.

[6] Hsu-Yaung, K., Jing-Shiuan, H. \& Chaur-Tzuhn Ch., Drought forecast model and framework using wireless sensor networks. Journal of Information Science and Engineering, 22, pp.751 - 769, 2006.

[7] Soloobera J., Detecting forest fires using wireless sensor networks with waspmote. $\underline{\text { www.libelium.com }}$

[8] Ning, $\mathrm{Xu}$ A, Survey of sensor network applications. www.niamwebs.com/sdb/a-survey-of-sensor-network applications.html

[9] Kershner, R., The number of circles covering a set. Amer. J. Mathematics, 61(3), pp. $665-671,1939$.

[10] Zhang, H. \& Hou, J.C., Maintaining sensing coverage and connectivity in large sensor networks. Ad Hoc \& Sensor Wireless Networks, 1(1-2), pp.89124, 2005.

[11] Kuznetsov, V. Yu., Tasks cover orthogonal polygons with forbidden areas. Journal of USATU, 10(2), USATU: Ufa, pp.177 - 182, 2008. 